\title{
LA ETNOBIOLOGÍA EN ÁREAS RURALES Y SU APORTE A LA LUCHA PARA DESENTRAÑAR SESGOS PATRIARCALES
}

\author{
ETHNOBIOLOGY IN RURAL AREAS AND ITS CONTRIBUTION TO THE STRUGGLE \\ TO UNRAVEL PATRIARCHAL BIASES
}

Ana Haydeé LADIO

${ }^{1}$ INIBIOMA (Instituto de investigaciones en Biodiversidad y Medio Ambiente, CONICET-UNComahue). Quintral 1250. 8400. S.C. de Bariloche. Argentina. email: ahladio@gmail.com

\section{RESUMEN}

Submitted: 07/05/2020; Accepted: 23/06/2020

El ecofeminismo ha cobrado importancia entre las lideresas campesinas aborígenes y no aborígenes de América Latina. Este enfoque plantea que la dominación extractivista que propone la lógica de mercado sobre la Naturaleza, es la misma que se imprime en los cuerpos de las mujeres desde hace siglos de colonización. Ambos, son territorios-cuerpos sujetos a relaciones de desigualdad y sometimiento. Los estudios rurales muestran que, bajo esa lógica neoliberal-patriarcal, solo se pondera el trabajo productivo que genera dinero realizado por hombres, dejando invisible el trabajo doméstico o el informal de las mujeres. Asimismo, permanece oculto su rol en la trasmisión de saberes y prácticas agropecuarias. En este artículo, deseo reflexionar sobre el papel de los trabajos etnobiológicos en visibilizar cuestiones de género. Propongo que la etnobiología ha tenido perspectivas compartidas con la aproximación ecofeminista. En sus evidencias empíricas y desarrollos teóricos ha desarmado supremacías propias de la lógica patriarcal, visibilizando el rol de las mujeres en la vida rural, especialmente cuando: 1- ha estudiado las distintas formas de conocimiento entre hombres y mujeres, 2- se ha centrado en el patrimonio material e inmaterial y los procesos de trasmisión cultural, 3- ha resaltado visiones locales de una Naturaleza no subordinada a la Cultura (por ende, al patriarcado), 4- ha visibilizado vínculos emocionales. Sin embargo, muchas veces los trabajos etnobiológicos no han sido suficientemente explícitos en desentrañar los sesgos patriarcales, por ende, se proponen seis ideas para desarticularlos, tanto de índole metodológico como conceptual. El carácter híbrido de la etnobiología provee de herramientas clave para dar cuenta del lugar multifacético de las mujeres en la conservación de la Naturaleza.

Palabras-clave: mujeres, ecofeminismo, diálogo horizontal, Naturaleza-Cultura, ética del cuidado.

\begin{abstract}
Eco-feminism has gained importance among indigenous and non-indigenous women farmers' leaders in Latin America. They argue that the extractive domination proposed by the logic of the market over Nature is the same that has been imprinted on women's bodies for centuries of colonization. Both are territories-bodies subject to relations of inequality and submission. Rural studies show that under this neoliberal-patriarchal logic, only productive work that generates money is considered, carried out by men, leaving women's domestic or informal work invisible. Moreover, their role in the transmission of agricultural knowledge and practices remains hidden. In this article, I wish to reflect on the role of ethnobiological work in gender studies. I propose that ethnobiology has shared perspectives with the ecofeminist approach. Empirical evidence and theoretical developments of ethnobiology have disarmed supremacy proper to patriarchal logic, making visible the role of women in rural life, specially when: 1) has studied the different forms of knowledge between men and women, 2) has focused on the material and immaterial heritage and processes of cultural transmission, 3) has highlighted local visions of a Nature not subordinated to Culture (therefore, to patriarchy), 4) has made visible emotional links. However, in many times the ethnobiology has not been sufficiently explicit in unraveling the patriarchal biases, therefore, six ideas are proposed to disarticulate them, both of methodological as conceptual nature. The hybrid character of ethnobiology provides key tools to account for the multifaceted place of women in nature conservation.
\end{abstract}

Keywords: ecofeminism, horizontal dialogue, Nature-Culture, ethics of care. 


\section{INTRODUCCIÓN}

Gran parte de los trabajos etnobiológicos desarrollados en áreas rurales tienen como premisa ética valorar los vínculos creados con plantas y animales que forman parte de su vida cotidiana (LADIO, 2017). En este sentido, muchos de los estudios han enfatizado el rol diferencial de mujeres y hombres en la construcción colectiva de saberes (KAINER y DURYEA, 1992; CAMOUGUERRERO et al., 2007; ALBUQUERQUE et al., 2011; ANDRADE et al., 2016). Es por eso que los temas de género han atravesado todos los hallazgos y conclusiones de la disciplina, aun sin proponérselo.

En este artículo, deseo reflexionar sobre el papel que han tenido los trabajos etnobiológicos en visibilizar cuestiones de género en el ámbito rural. Propongo, como eje de discusión, la idea que la etnobiología comparte perspectivas comunes con la propuesta ecofeminista, sin embargo, es necesaria una mayor discusión teórica y/o metodológica para no caer en sesgos patriarcales. Es decir, no convalidar la creencia que fomenta que las diferencias de orden biológico que existen evidentemente entre mujeres y hombres justifican desigualdades en la vida campesina.

\section{Estudios rurales y ecofeminismo}

Debido a la presión de numerosos colectivos feministas en todo el mundo, se ha puesto de manifiesto con datos empíricos que las relaciones de poder que se dan entre los géneros son en general favorables a los varones y discriminatorias para las mujeres (CABEZAS GONZÁLEZ, 2015). Esta relación de poder atraviesa todo el entramado social-cultural, y se intersecta con cuestiones de clase social, etnia, edad, ruralidad-urbanidad, etc. (TRPIN et al., 2017).

El ámbito rural se ha convertido en un escenario crucial para analizar dichas relaciones de género por diversas razones. Viu et al. (2010) atribuyen al ámbito rural un conservadurismo marcado que genera fuertes desigualdades de género persistentes hasta el día de hoy. Por otro lado, los ámbitos rurales se ven afectados en la actualidad por la creciente importancia de actividades no agrícolas como las artesanías, las manufacturas de materias primas, el comercio y el turismo; la mayor fluidez de intercambio con las ciudades y lo global, que generan cambios culturales significativos, principalmente porque las mujeres participan cada vez más en estas actividades (CARNEIRO, 1994; KAY, 2013).

Los estudios rurales en los últimos años han reflejado fuertes discusiones y cambios conceptuales debido a la importancia que ha ejercido el movimiento feminista en las múltiples esferas del mundo científico y no científico (KAY, 2013). Diversas autoras han mostrado cómo el ecofeminismo ha cobrado importancia entre las lideresas campesinas aborígenes y no aborígenes de distintas partes de América Latina (PULEO, 2015; PENA, 2017).

La aproximación ecofenimista, en sus distintas versiones, ha podido cristalizar y poner en discusión la idea que la dominación extractivista que propone la lógica de mercado capitalista sobre la Naturaleza es la misma que se imprime en los cuerpos de las mujeres desde hace siglos de colonización (SILIPRANDI, 2010; ALONSO y DÍAZ 2018; KLIER y NÚÑEZ, 2019). Estos territorios-cuerpos están sujetos a relaciones de desigualdad y sometimiento con el objetivo de producir más ganancias (CRUZ HERNÁNDEZ, 2016; NAVARRO TRUJILLO y GUTIÉRREZ AGUILAR, 2017). Por otra parte, las principales corrientes del ecofeminismo denuncian que al identificarse dualmente a la mujer con la naturaleza salvaje, indómita, y al hombre, con lo racional, la cultura, se introduce una jerarquía que sirve de justificación para la opresión (KLIER y NÚÑEZ, 2019).

Es importante destacar que entre las distintas visiones del ecofeminismo, el llamado ecofeminismo clásico, efectivamente pone énfasis en el rol femenino en el mantenimiento de las redes de la vida. Según esta visión las mujeres han establecido desde hace milenios una relación estrecha e ineludible con la Naturaleza construyendo una ética de cuidado singularmente femenina (SHIVA, 1991; PULEO, 2015; ULLOA, 2016).

Sin embargo, otras vertientes, como la del ecofeminismo constructivista, se centran en desactivar estas relaciones de "lo femenino" o "virtudes de género", considerándolas categorías esencialistas (SILIPRANDI, 2010; PENA, 2017). Según esta posición, este rol asignado a las mujeres como cuidadoras es una consecuencia de la rígida jerarquía patriarcal. Según varias autoras del libro compilado por Puleo (2015), la dominación masculina ha relegado a la mujer al espacio privado e emocional, alejándola de los centros de decisión y restringiéndola al ámbito doméstico. Se ha 
construido un sentido común asociando a la mujer con lo natural y lo familiar, que inclusive ha sido fuertemente adoptado por los Estados latinoamericanos a modo de disciplinamiento (PÉREZ, 2019).

Para el movimiento ecofeminista, buscar soluciones contra el androcentrismo implica encontrar soluciones para la sustentabilidad socio-ambiental (PULEO, 2015). Por ende, sus principales premisas son la equidad de género y la protección del medio ambiente, entendiendo que una sociedad sin explotación de las mujeres, es también una sociedad sin explotación de la naturaleza (SILIPRANDI, 2010). Muy especialmente trata de fomentar la universalización de la ética del cuidado, que incluye el compromiso de tanto mujeres como hombres. Estos propósitos solo pueden ser realizados a través de un diálogo horizontal e intercultural de saberes (PULEO, 2015).

Los estudios rurales muestran que bajo la lógica neoliberal-patriarcal arriba señalada, solo se pondera el trabajo productivo que genera dinero, principalmente realizado por hombres, dejando invisible el trabajo doméstico o el informal llevado a cabo principalmente por las mujeres (TRPIN et al., 2017). En algunos casos, las evidencias de una mayor incorporación de la mujer en ferias o mercados de artesanías, solo le han significado una jornada doble de trabajo para ellas, ya que los hombres generalmente no han aumentado significativamente su participación en las actividades del hogar (ALONSO y TRPIN, 2018). Asimismo, según las especialistas, permanece oculto el rol significativo de las mujeres en la trasmisión de saberes y prácticas agropecuarias, vitales para la reproducción de los modos de vida comunitarios. A diferencia de lo que sucede con las liderezas campesinas, que en realidad no son una regla, sino una excepción en el ámbito rural, la mayoría de las mujeres rurales sufren en su día a día la deslegitimación de sus tareas y derechos (SILIPRANDI, 2010).

\section{¿Cuáles son las perspectivas compartidas entre la etnobiología y el ecofeminismo?}

Se puede pensar que la etnobiología de los últimos años, considerando sus evidencias empíricas y desarrollos teóricos, ha colaborado en la reflexión tendiente al desarmado de supremacías propias de la lógica patriarcal. Si bien los trabajos etnobiológicos no tienen, en general, una postura epistémica crítica de la realidad, han visibilizado el rol de las mujeres en la vida rural, sus habilidades y capacidad de manejo en la gestión de la Naturaleza (KAINER y DURYEA, 1992). Además, ha dado cuenta de su rol primordial en la alimentación y la soberanía alimentaria y de su escaso reconocimiento (TASSI y BEZERRA, 2020). Nuestra disciplina fuertemente ha señalado la importancia de incluir a la voz de las mujeres en proyectos de desarrollo forestal o de conservación para que las acciones sean más efectivas e igualitarias (WESTERMANN et al., 2005; TOLEDO y ALARCÓN-CHÁIRES, 2018).

Tomando ideas de Velasco Sesma (2015) sobre las características que poseen los sistemas de opresión que son denunciados por el ecofeminismo, nuestra disciplina tiene justamente proposiciones opuestas a sistemas opresivos. La etnobiología aboga por un pensamiento no jerárquico-valorativo de los distintos tipos de conocimientos sobre plantas y animales evitando nociones de superioridad e inferioridad, por ejemplo, del sistema científico versus los sistemas de conocimientos locales (LADIO, 2017). También discute aproximaciones que implican dualismos valorativos y no plurales como pares opuestos excluyentes en los que una de las partes se considera más valiosa que la otra (DELGADO y ESCÓBAR, 2006). Por otra parte, ha cuestionado la lógica neoliberal materialista y productivista que ha desencadenado la pérdida de diversidad biocultural (TOLEDO y BARRERAS-BASSOLS, 2008).

A partir de una mirada amplia (pero no exhaustiva) de las investigaciones etnobiológicas, se pueden distinguir focos de estudio que serían particularmente relevantes para el ecofeminismo e implican visiones compartidas: 1- El estudio de las distintas formas de conocimiento sobre los elementos de la Naturaleza entre hombres y mujeres en las diversas sociedades del mundo, 2- El estudio de lo inmaterial en los patrimonios bioculturales como contraposición a una visión únicamente materialista, 3-Los estudios de la relación Naturaleza-Cultura distinguiendo aquellas sociedades en donde la Naturaleza no está separada ni subordinada a la Cultura (por ende no estaría subordinada a los dictámenes del patriarcado) y 4- Los estudios que ha visibilizado valores emocionales en relación a plantas y animales presentes tanto en mujeres y hombres (Figura 1).

\section{El estudio de las diferentes formas de conocimiento entre hombres y mujeres visibiliza roles de género}

Los trabajos etnobiológicos han tomado reiteradamente la temática de los saberes y prácticas diferenciales entre mujeres y hombres sobre plantas y animales (TORRES-AVILEZ et al., 2016). Se 
han detallado similitudes y diferencias entre géneros en la manera de conocer, utilizar y conceptualizar a los bienes naturales en las comunidades rurales (CANIAGO y SIEBERT, 1998; VIU et al., 2010). Estos trabajos han podido contrarrestar miradas homogeneizantes de las culturas y evidenciar roles de género (BEARDSWORTH et al., 2002; VAZQUEZ-GARCIA, 2007).

Los intereses y motivaciones en el uso de recursos naturales pueden ser muy distintos entre géneros y culturas (ATRAN et al., 2004). Los estudios de caso muestran que las generalidades no existen y que el rol de las mujeres y hombres en la gestión ambiental depende fuertemente de las circunstancias locales. Por ejemplo, en un estudio en cuatro comunidades de México sobre las habilidades y conocimientos agropecuarios de las mujeres con respecto a los hombres, mostró que depende fuertemente de las condiciones geográficas y/o culturales (BAIN, 1993).

La diversa literatura ha mostrado también cómo los cambios socio-ambientales en las comunidades son factores que atraviesan las cuestiones de género y que complejizan aún más su interpretación (MAI et al., 2012). Por ejemplo, en África, se encontraron pocas diferencias entre hombres y mujeres en relación a conocimientos sobre plantas comestibles, para construcción y medicinas, interpretado por los autores como una consecuencia de la emigración de los hombres que han debido irse de las áreas rurales para buscar alternativas económicas y entonces las mujeres quedaron a cargo de todas las actividades de la finca (KRISTENSEN y BALSLEV, 2003).

Otro punto importante es que los trabajos etnobotánicos han permitido visibilizar el trabajo de las mujeres en actividades agroforestales que normalmente han sido descriptas como masculinas. Por ejemplo, el trabajo clásico de Fortmann and Rocheleau (1985) en Kenia, India y Republica Dominicana que desmitifica que ellas no son "simples amas de casa" (textual) y que realizan actividades cruciales en la selección y cuidado de especies para la reforestación, participan en movimientos de defensa de los bosques y son las encargadas de organizar grupos efectivos de trabajo para la ejecución de proyectos agroforestales.

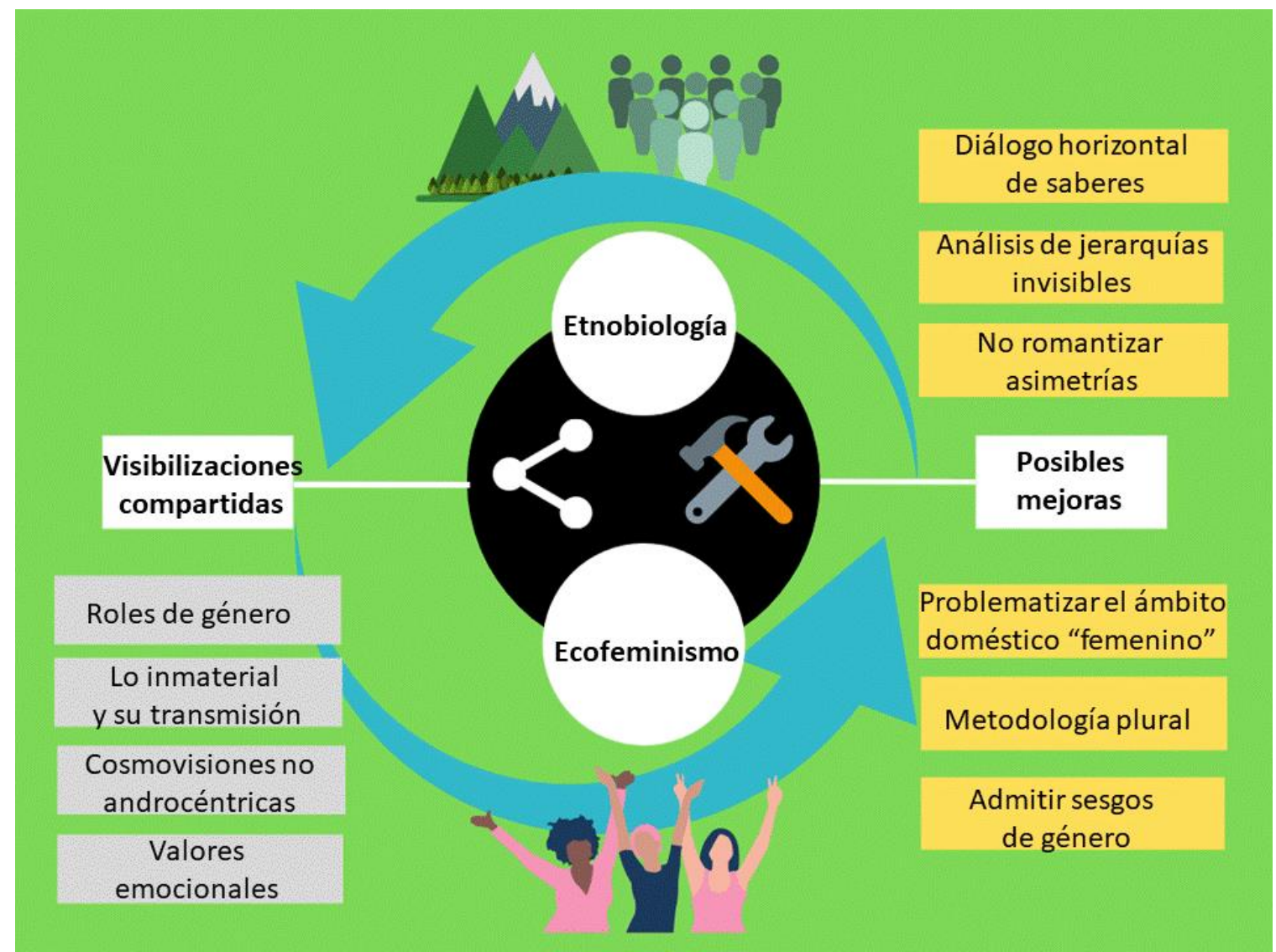

Figura 1. La etnobiología y el ecofeminismo se encuentran en un proceso de interacción y desarrollo permanente. Muchos de los trabajos actuales abordan temáticas que son importantes para el ecofeminismo (visibilizaciones compartidas). Sin embargo, es posible perfeccionar nuestros estudios para no caer en sesgos patriarcales (posibles mejoras). 
Además, los trabajos etnobotánicos han podido mostrar el rol de las mujeres en la comercialización de recursos (normalmente asignada a los hombres) como es el caso de la venta de frutos de asaí (Euterpe precatoria Mart.) en la amazonia colombiana, en donde el $70 \%$ es llevado a cabo por mujeres (ARANGUREN et al., 2014). O en el caso los tejidos de lana de oveja (Ovis orientalis aries L.) que en varias regiones de la Patagonia son comercializados por mercados gestionados de manera cooperativa por mujeres campesinas (CARDOSO et al., 2015). O el caso de la venta de hongos silvestres en México que es una actividad llevada a cabo principalmente por mujeres (GARIBAY-ORIJEL et al., 2012).

\section{El estudio de lo inmaterial en los patrimonios bioculturales fortalece una mirada no patriarcal y visibiliza el rol de las mujeres en la transmisión social del conocimiento}

Los estudios etnobiológicos sobre patrimonio han resaltado la necesidad de considerar lo tangible y lo intangible (MAFFI, 2005). El patrimonio biocultural de un lugar es el resultado de la compleja interacción entre la cultura y el ambiente a lo largo del tiempo que ha dado como propiedad emergente a la diversidad biocultural. Esta no solo incluye al conjunto de variedades vegetales, razas animales, especies silvestres, espacios, sistemas y paisajes intencionalmente creados, mantenidos y utilizados por grupos culturales, sino también refiere a las dimensiones epistémicas y linguísticas, es decir las creencias, los rituales, las tradiciones, el arte, las reglas de uso del territorio, los conocimientos y las innovaciones desarrolladas a lo largo del tiempo, tanto por hombres como mujeres (TOLEDO y ALARCÓN-CHÁIRES, 2018).

Dicho foco de estudio amplía la mirada hacia los bienes naturales que no pueden simplemente cuantificarse en dinero y que son parte de una herencia cultural fundamental. Los poemas, cantos y mitos recogidos por distintas investigaciones en pueblos originarios (AILLAPAN y ROZZI, 2004; SUÁREZ, 2009), las técnicas y saberes llenos de espiritualidad que contribuye con uso sustentable de la Naturaleza (GARIBALDI and TURNER, 2004; GARIBALDI, 2009; KUSHWAH et al., 2017). Asimismo, las distintas expresiones pictóricas y artísticas que revelan el sinfín de significados que tienen los organismos vivos para los humanos, más allá de su uso material (COSTA NETO et al., 2009).

En este sentido, se ha asignado un rol fundamental generalmente a las mujeres en la trasmisión social de conocimientos ligados a la Naturaleza, particularmente de plantas hortícolas (EYSSARTIER et al., 2008), plantas medicinales (VIEIRA y MILWARD-de-AZEVEDO, 2018) y plantas silvestres (OCHOA y LADIO, 2015). También, las mujeres han sido señaladas como las encargadas del legado de saberes familiares o comunitarios, actuando como verdaderos reservorios (VOEKS, 2007). Por ejemplo, de rezos, mitos y cantos sagrados que son parte de la herencia biocultural mantenida por las mujeres (LENAERTS y SPADAFORA, 2008; REYES-GARCÍA et al., 2009; ZENT, 2014; ATHAYDE et al., 2017). Es el caso en la cultura Qom, que las mujeres deben invocar al dueño de las especies silvestres para justificar la necesidad de recolección para no despertar su ira (LENAERTS y SPADAFORA, 2008).

Todo esto, desde un enfoque ecofeminista podría significar la revalorización de las acciones de las mujeres en aspectos sustanciales de la vida en sociedad y que al mismo tiempo pone en tela de juicio la sobrevaloración de las acciones meramente de valor económico material.

\section{El estudio de la relación Naturaleza-Cultura permite distinguir cosmovisiones no androcéntricas}

Gran parte de la literatura latinoamericana se ha dedicado a distinguir fuertemente las cosmovisiones ligadas al antropocentrismo que separan a la Naturaleza de la Cultura para poder explotarla, de aquellas cosmovisiones biocéntricas o relacionistas presentes en las culturas originarias (MAFFI 2005; LADIO 2017). En estas visiones de tipo relacionista, que no separan a la Naturaleza de la Cultura, el concepto de territorio es esencial porque es vivenciado como el lugar indispensable para el desarrollo de la vida de hombres y mujeres. En concordancia con Alonso y Trpin (2018), los cuerpos y los territorios están íntimamente relacionados entre sí en las sociedades, por eso son parte de las luchas ecofeministas. En este sentido, los etnobiológos y etnobiológas hemos demostrado que la única manera que las comunidades tradicionales y locales tienen para proveerse de alimentos sanos y medicina es a través del acceso a territorios bien conservados y sin contaminación. No es casual que este principio forme parte de nuestro Código de ética como parte fundamental de nuestro enfoque profesional (VILLAMAR et al., 2018). 
Los estudios también han mostrado que las miradas relacionistas asocian al territorio con procesos de crianza mutua en donde los seres humanos participan activamente en la construcción de los paisajes y son también los humanos criados por los otros seres del paisaje (LEMA, 2013). En ese sentido, la palabra criar no fue asignada por los etnobiólogos o etnobiológas a un "valor femenino" sino a la responsabilidad humana de cuidar y regenerar la tierra en sus múltiples espacios bioculturales.

En este sentido, la etnobiología han podido desenmascarar la inexistencia de una forma única de percibir a la Naturaleza y gestionarla (TOLEDO y ALARCÓN-CHÁIRES, 2018), mostrando modelos alternativos a la sobreexplotación y dominación de la sociedad de mercado patriarcal. Ha señalado la importancia de poner en acción a las éticas bioculturales de los pueblos originarios con la Naturaleza, en un marco de relación en donde los distintos actores sean concebidos de manera igualitaria como cohabitantes con los mismos derechos y obligaciones para hombres y mujeres (ZENT, 2014; ROZZI, 2016).

\section{El estudio de valores emocionales permite desactivar miradas sexistas}

La etnobiología ha visibilizado fuertemente la importancia de considerar lo emocional en la relación humanos naturaleza (AIGO y LADIO, 2016). Muchos artículos han adherido a las ideas del "sentipensar" de Arturo Escobar (2016) o la de revalorizar la emoción como dice Enrique Leff (2004). De alguna manera, la disciplina en algunos autores no ha renunciado a caracterizar las emociones y sentimientos de los seres humanos hacia la Naturaleza como un elemento relevante para la conservación (ROZZI, 2012). Dado que la reivindicación ecofeminista busca recuperar, tanto para hombres y mujeres, el sentido de una emotividad perdida frente a la mayor valorización de lo racional y material, es aquí donde los trabajos cobran una vital importancia en mostrar cómo los vínculos con la Naturaleza están nutridos de sentimientos y emociones en ambos géneros. Esto se ha visto reflejado en estudios acerca de los vínculos con animales (NOLAN et al., 2001; BARRERA-BASSOLS y TOLEDO, 2005; VARGAS-CLAVIJO et al., 2010; HERRMANN et al., 2013; CARVALHO et al., 2018) pero también con las plantas (HUNN, 2014; VÁRI et al., 2020). Vári et al. (2020) han comparado entre hombres y mujeres la citación de valores emocionales en la recolección de plantas medicinales y silvestres, y evidenciaron que ese aspecto es igualmente importante en ambos géneros, desmitificando miradas sexistas sobre la emoción.

También, la literatura etnobiológica que se ha dedicada a la salud indígena y ha dado innumerables ejemplos de la importancia del concepto de bienestar físico, emocional y espiritual para las sociedades (SHAHID et al., 2010; TORRI, 2010), y de no solo atender a los componentes materiales del sistema médico (LENAERTS y SPADAFORA, 2008). Muy especialmente destaco cómo se ha documentado sobre la alegría que da tener flores en los huertos en distintas partes del mundo y cómo este sentimiento experimentado es el impulsor del enriquecimiento de especies en dichos espacios (GASCO, 2008, CARDOSO et al., 2015; FURLAN et al., 2017).

\section{¿CÓMO EVITAR LOS ESTEREOTIPOS DE GÉNERO EN LA INVESTIGACIÓN ETNOBIOLÓGICA?}

Si bien, Vieira y Milward-de-Azevedo (2019) hablan de la importancia de los trabajos etnobiológicos para validar el rol significativo de las mujeres en las áreas rurales, no podemos negar que en cierta parte de la literatura han primado visiones materialistas, basadas en categorías binarias, dicotómicas y sexuadas (LADIO y LOZADA, 2004). Con intención o no, hemos convalidado un modelo que sostiene los esterotipos de género, siendo inclusive, contraproducentes para propiciar cambios en las comunidades locales y en la implementación de políticas públicas. Hace falta prestar mayor atención en ciertos aspectos que pueden servir para mejorar nuestras investigaciones. A continuación, incluyo seis puntos posibles (ver Figura 1).

\section{Propiciar un verdadero diálogo horizontal de saberes}

Numerosos artículos etnobiológicos han mostrado la existencia de estructuras de poder que han deslegitimado o desconsiderado a los saberes comunitarios sobre la Naturaleza, excluyéndolos habitualmente de ámbitos de decisión. En consecuencia, un axioma generalizado en los trabajos ha sido el de fomentar el diálogo horizontal de saberes entre integrantes de una comunidad con los investigadores, técnicos y decisores involucrados en la gestión de la Naturaleza (RUIZ PÉREZ y ARGUETA VILLAMAR, 2011). Sin embargo, estos mismos esquemas de poder se repiten en el 
interior de las comunidades rurales y en consecuencia las mujeres suelen quedar relegadas (SILIPRANDI, 2010).

El diálogo al interior de las comunidades entre hombres y mujeres poco se ha estudiado desde la etnobiología. La democratización de la palabra y la inclusión de las mujeres en esferas movidas por la lógica de acumulación y patriarcado no son fáciles. En concordancia con Fortmann and Rocheleau (1985), esta tarea necesita de arreglos especiales que puedan incentivar a la participación femenina desde otro lugar. En algunos casos será necesario separar a las mujeres especialmente de ámbitos opresivos con el objetivo de que puedan expresarse libremente. También será necesario aliviar temores a los varones y las mujeres sobre estas actividades junto a ellas para que no se sientan amenazados en su labor cotidiana. Además, tomado como ejemplo el trabajo de (ALONSO y DÍAZ, 2018), deberíamos armar equipos entre científicas y mujeres campesinas de modo articular más efectivamente un diálogo horizontal en donde se visibilicen sus problemáticas y se generen propuestas emancipadoras.

\section{Analizar jerarquías imperantes e invisibles en las relaciones de género}

Pocas investigaciones etnobiológicas ha podido, desde mi punto de vista, explicitar y discutir los orígenes fundantes de los roles de género en la mayoría de los estudios de caso en el ámbito rural. Por ejemplo, Viu et al. (2010) sugieren al encontrar mayor conocimiento de plantas medicinales entre campesinas de Goiás (Brasil) que entre hombres, que se debe al conservadurismo patriarcal rural, aunque sin dar mayores profundizaciones de los mecanismos operantes. Kai et al. (2014), por otra parte, encuentran que hombres y mujeres identifican diferencialmente aves y mamíferos pero solo se limitan a relacionarlo con cuestiones de división de trabajo. En general, el máximo despliegue en la discusión de los trabajos etnobiológicos es decir que las diferencias se deben a roles determinados culturalmente y no se prosigue con interpretaciones o reflexiones posteriores (ej. BAIN, 1993).

Una excepción interesante es el caso de Goebel (1998) quien indica que la recolección de plantas leñosas pertenece a la esfera masculina, pero no como consecuencia de una división de labor, sino a una expresión de poder sobre las mujeres. Resulta entonces importante pensar en las jerarquías existentes, no solo en las diferencias. Un trabajo sobre horticultura familiar en México muestra que las mujeres tienen un rol muy significativo en la producción de alimentos, pero que a la hora de comer, los hombres comen primero que mujeres y niños, mostrando jerarquías de poder que podrían tener consecuencias nutricionales (BAIN, 1993), Este patrón de que las mujeres comen después de los hombres, y menos, se ha encontrado también en Nepal (BYERS y SAINJU, 1994).

Es interesante el trabajo de Ayantunde et al. (2008), quienes comparan el conocimiento de plantas útiles entre mujeres casadas y no casadas, situación que no es a menudo tratado en las investigaciones etnobiológicas, invisibilizando un aspecto clave en la vida de las mujeres rurales. Según los autores, se encontró que las mujeres casadas saben más sobre plantas debido a su mayor edad promedio y a su mayor experiencia doméstica. En futuros trabajos deberemos, por ejemplo, poner a prueba si es visualizado por las campesinas y los campesinos un orden jerárquico en la posesión de conocimientos y en la ejecución de prácticas sobre los recursos naturales que explique las desigualdades a veces existentes. Poder generar preguntas que diferencien entre las personas que se encargan del cuidado, y el verdadero propietario/a del recurso, preguntar por quienes toman las decisiones, y si se han producido cambios en estos aspectos en el tiempo.

\section{No romantizar a las asimetrías como virtudes femeninas}

La obra de Alicia Puleo (2015) nos invita a la reflexión sobre las diferencias de género encontradas en una comunidad para pensarlas como asimetrías que surgen como consecuencia a limitaciones impuestas por la exclusión patriarcal.

Para muchas autoras ecofeministas estructuralistas, la fuerte construcción social existente acerca de las mujeres "cuidadoras" de las personas y de los demás seres, estarían reflejando estereotipos normalizantes sobre cómo deberían ser hombres y mujeres. En muchos estudios de caso etnobotánicos se halagan las virtudes femeninas de cuidado (EYSSARTIER et al., 2015). Si bien, representan descripciones de la realidad, su formulación sin cuestionamiento a las posibles razones que operan en dicha sociedad, podría obstaculizar el avance de propuestas superadoras.

Price y Ogle (2012), en sus estudios sobre recolección de plantas silvestres en Asia, discuten, por ejemplo, que no pueden responder por qué las mujeres predominan como recolectoras, pero que sin duda es el reflejo de racionalidades socioculturales impuestas. Su trabajo muestra que las mujeres 
cada vez deben recorrer distancias más largas, y en condiciones más desfavorables para encontrar plantas silvestres debido a la deforestación. Las mujeres son entonces las primeras que sufren la destrucción ambiental, la perdida de conocimientos y prácticas tradicionales dado que es la esfera de acción socialmente establecida para ellas (BYERS y SAINJU, 1994). La romanización de este esfuerzo, y la de otros, como el de su responsabilidad como cuidadoras de huertos, ha sido altamente señalado en los trabajos (HOWARD, 2006). Sin dudas, la huerta es un espacio femenino de gran importancia donde las mujeres pueden gestionar intercambios fortaleciendo redes sociales indispensables, entre otras actividades sustanciales de la vida familiar y la alimentación. Este rol también les otorga prestigio y reputación a las mujeres, que como beneficio adicional quizás le agregue una mejor posición de negociación con los hombres. Pero también, puede ser un espacio de neta subordinación al modelo patriarcal, es decir el único espacio de interacción posible. Repensar junto con las campesinas la superación a estas posibles situaciones si existiesen, cuestionando la reivindicación de estas tareas como virtudes de género sería un gran avance para nuestros estudios.

\section{Problematizar el espacio doméstico, lo más cercano, es "femenino"}

Los trabajos etnobiológicos han mostrado que hombres y mujeres poseen asignaciones de uso del espacio diferenciales, y que dependen fuertemente de cada contexto cultural. Se observa una tendencia a asociar mayor conocimiento o uso de plantas y animales de ámbitos cercanos peridomésticos entre las mujeres que entre los hombres (KAINER y DURYEA, 1992; LADIO y LOZADA, 2004; ALBUQUERQUE et al., 2011). Estos resultados por lo señalado arriba, pueden ser indicativo de estructuras patriarcales que restringen el accionar femenino a los espacios cercanos a la casa, por lo que necesitamos una discusión más profunda de estos aspectos.

Fadiman (2005), en una comparación de los espacios usadas para la gestión hortícola entre una comunidad de colonos e indígenas de Ecuador, encontró que las mujeres colonas gestionan los huertos más cercanos que en el caso de las indígenas. También, establece que cuanto más ganado tiene la finca, los hombres pasan más tiempo en su cuidado y la mujer queda atendiendo mayormente quehaceres domésticos en la casa, y que cuando más tecnificada está la actividad agropecuaria ese patrón se agudiza aún más.

En este mismo sentido, Reyes-García et al. (2010) han encontrado en zonas montañosas de la Pla. Ibérica (España), que los huertos manejados por hombres son más grandes y más alejados de las casas que los manejados por mujeres. Los autores lo asocian a que las mujeres al tener que compartir otras actividades domésticas eligen los huertos domiciliarios (textual), en cambio los hombres tienen más libertad de usar las tierras agrícolas (textual), inclusive las tierras más alejadas. En este trabajo se evidencian posibles problemáticas subyacentes, y se insta a la inclusión futura de datos sobre derecho y acceso legal de la tierra de las mujeres como un factor explicativo importante, dado que las mujeres no parecen tener un acceso igualitario.

Otro trabajo interesante que presenta aspectos poco desarrollados en los estudios etnobiológicos es el de Lenaerts y Spadafora (2008), junto a mujeres Quom en el que se señala que si bien las mujeres son las principales conocedoras y recolectoras de plantas útiles, las mujeres jóvenes no salen al monte debido al temor de violencia sexual llevada a cabo por varones criollos quienes son los que han usurpado sus tierras.

De acuerdo a Fadiman, (2005), en los estudios de género es indispensable diferenciar el uso de espacios ligados al género, además de las actividades asociadas a género dado que puede brindar información muy rica de análisis. En este sentido, es interesante lo que propone Voeks (2007) que establece que los hombres en general poseen mayor conocimiento de recursos de áreas naturales no disturbadas, mientras que el conocimiento de las mujeres está fuertemente asociado a ambientes antropogénicos, los cuales pueden estar lejos o cerca de la casa.

Todos los casos arriba señalados pueden servirnos de ejemplo para poder problematizar la complejidad de factores que fuerzan a las mujeres a permanecer cerca de las casas, particularmente asociadas a las propias trayectorias de vida en condiciones de desigualdad.

\section{Mejorar la pluralidad en la metodología}

Las investigaciones etnobiológicas pueden mejorar su metodología para evitar la invisibilización de las acciones y miradas de las mujeres. Según Vazquez-Garcia (2007), varios trabajos han ignorado el rol de las mujeres, muchas veces ni en la metodología aparecen referencias de 
género de los entrevistados que participan en los estudios. Esto debiera ser desalentado como forma de evitar miradas homogeneizantes de los integrantes de una comunidad.

Sin embargo, varios autores han pensado en cómo mejorar sus investigaciones con mujeres. Por ejemplo, en un estudio de huertos domésticos en los Alpes austriacos, se sugiere que sean mujeres etnobotánicas las que realicen el trabajo de campo dado que la mayoría de las horticultoras son mujeres (VOGL et al., 2004). En cambio, Reyes-García et al. (2010) presentan un método alternativo para evitar dicho sesgo en la evaluación de huertos. Los investigadores indican que ellos seleccionan por huerto, no por informante, y que luego lo clasifican como espacio femenino, masculino o mixto de acuerdo con la predominancia de tareas de cada género e independientemente de quien contestó el cuestionario. También Lenaerts y Spadafora (2008) destacan la importancia de haber conversado con las mujeres lejos de la presencia masculina en la comunidad Ashéninka (Amazonas), así las mujeres pudieron desplegar amplios conocimientos de los "secretos" masculinos y femeninos asociados al uso de plantas medicinales.

Otro gran cuestionamiento metodológico destacado por Mai et al. (2012) es el uso de entrevistas a la persona a cargo del hogar como representante de una unidad familiar. Este acto puede generar la exclusión automática de la participación de las mujeres. Otro problema es asumir, sin evidencia empírica, que la unidad doméstica es igualitaria en la distribución de ingresos y/o en el uso de los recursos naturales. Los hogares pueden ser el lugar de una competencia intensa sobre recursos económicos y naturales que debe ser mayormente diferenciada. Una posibilidad es preguntar a las mujeres por estas asimetrías en la unidad familiar, y por las actividades que le fueron asignadas culturalmente a cada uno de los géneros, y qué disyuntivas, deseos encontrados o conflictos eso les genera según su perspectiva.

Por el contrario, Edwards et al. (2005) han señalado el efecto inverso en el estudio sobre el uso de plantas medicinales, cuando solo se encuentra a las mujeres en la casa y ellas responden en representación de toda la unidad familiar. Prestar atención a la pluralidad de nuestra metodología considerando la multidimensionalidad de los saberes y prácticas entre hombres y mujeres es sustancial para entender verdaderamente los procesos bioculturales.

\section{Admitir que nuestros estudios tienen sesgos de género}

Es un hecho que nuestras investigaciones han arrastrado sesgos de diversa índole, que sin duda pudieron afectar negativamente a una visión de género adecuada. Como primera medida es aceptar la parcialidad de la propia visión y reflexionar sobre nuestros propios procesos de opresión o exclusión como investigadoras e investigadores (TRPIN et al., 2017). Otro tópico resaltado por estas autoras es la reflexión sobre el sesgo hetero-normativo en nuestras interpretaciones, al situar a la familia nuclear heterosexual como la norma para entender los procesos de la vida rural y dejando de lado otros modelos de convivencia posibles, por ejemplo, el hecho que muchas mujeres son jefas de hogar, u otras alternativas de familia.

Es llamativo que la literatura etnobiológica no se haya percatado de estas situaciones en sus publicaciones hasta ahora, siempre las familias son presentadas de manera uniforme. Es importante prestar atención a la visión del concepto de familia como un todo integrado y homogéneo que actúa como un bloque igualitario (RICHERI et al., 2013), dado que la realidad puede ser muy distinta, y sus integrantes pueden estar experimentado asimetrías que deben ser analizadas en mayor detalle.

También, debemos atender posibles sesgos clasistas con las mujeres en las investigaciones en áreas rurales, cuando se evalúa a la economía informal como una situación no especialmente contributiva. Muchas veces en los análisis socioeconómicos del uso de recursos naturales, se distingue diferencialmente al trabajo remunerado del no remunerado como una categoría de interés (LENAERTS y SPADAFORA, 2008). Una mayor valorización de lo remunerado puede ocasionar una visión errada del papel de las mujeres en la economía del hogar que debe ser remediada incrementando la multidimensionalidad de este tipo de variables.

Por último, me interesa señalar lo dicho por Trpin et al. (2017), en cuanto al trabajo de campo de las científicas en áreas rurales, pero trasferido al trabajo específico de las etnobiólogas. Nuestros pasos en el trabajo de campo, en los predios productivos rurales o en las áreas naturales, acarrean marcas de género; marcas de clase, y de otros tipos de marcas que no debemos olvidar y que actúan como sesgos para la interacción con las personas. Problematizar este aspecto, por ejemplo, en ámbitos hegemonizados por hombres, como son los ámbitos productivos en general, o también en aquellos netamente femeninos, puede darnos una oportunidad para interpretar mejor las subjetividades de los 
discursos de las mujeres y de los hombres. Y también es una oportunidad para escucharnos más a nosotras mismas a la hora de dar sentido a sus palabras.

\section{CONCLUSIONES}

La etnobiología, por su naturaleza conceptual-metodológica interdisciplinaria que integra lo social y lo biológico, tiene un rol crucial para visibilizar el lugar multifacético de las mujeres y hombres en la conservación de la Naturaleza. La etnobiología como la investigación feminista es contextual, experiencial y multi-metodológica, aspectos que le otorgan gran profundidad de análisis. Sin embargo, muchas veces los trabajos etnobiológicos no ha sido suficientemente explícitos en desentrañar las jerarquías patriarcales que operan en los cotidianos de las sociedades de estudio, y que también existen entre los propios investigadores e investigadoras. La importancia que ha adquirido la temática de género en la etnobiología puede llevar a la disciplina a un nuevo estadio de desarrollo si se logra un proceso de mayor reflexión sensible considerando algunos aspectos señalados en este texto. De esta manera, nuestros estudios pueden ser útiles en la implementación de políticas públicas tendientes a minimizar las desigualdades de poder, en especial para el caso de las mujeres campesinas.

\section{AGRADECIMIENTOS}

Primeramente, un fuerte agradecimiento a todas y todos los agricultores familiares del Noroeste de la Patagonia con quienes he aprendido sobre el amor a la tierra y a todos sus habitantes humanos y no humanos. A L. Laborda por compartir bibliografía feminista conmigo y a los dos revisores que han evaluado este trabajo. Por último, agradezco a Taline Cristina da Silva por incentivarme a pensar en el rol de las mujeres en la etnobiología y en la ciencia. Especial agradecimiento al Consejo Nacional de Investigaciones Científicas y Técnicas de la Argentina, al INIBIOMA y a la Universidad Nacional del Comahue.

\section{REFERENCIAS}

AIGO, J.; LADIO, A. Traditional Mapuche ecological knowledge in Patagonia, Argentina: Fishes and other living beings inhabiting continental waters, as a reflection of processes of change. Journal of Ethnobiology and Ethnomedicine, v. 12, n. 56, p. 1-17, 2016.

AILlAPAN, L.; ROZZI, R. Una etno-ornitología Mapuche contemporánea: veinte poemas alados de los bosques nativos de Chile. Ornitología Neotropical, v. 15, p. 1-16, 2004.

ALBUQUERQUE, U. P.; SOlDATI., G. T.; SIEBER, S. S.; RAMOS, M. A.; DE SÁ, J. C.; DE SOUZA, L. C. The use of plants in the medical system of the fulni-ô people (N Brazil): a perspective on age and gender. Journal of Ethnopharmacology, v. 133, p. 866-873, 2011.

ALONSO, G.; DÍAZ, R. Cuerpo y territorio desde lo alto de una torre : visibilidad, protagonismo y resistencia de mujeres mapuce contra el extractivismo. In: GÓMEZ, M.Ñ.; SCIORTINO, S. (ed.). Mujeres indígenas y formas de hacer política: un intercambio de experiencias situadas entre Brasil y Argentina. Temperley: Tren en Movimiento, 2018. p. 27-58.

ALONSO, G.; TRPIN, V. Territorios y cuerpos en el norte de la Patagonia: desafíos teóricos y metodológicos en tiempos de extractivismo. Revista de Estudios Marítimos y Sociales, 2018. Disponible en: URL: https://estudiosmaritimossociales.org/archivo/rems-13/dossier-alonso-trpin/ Acceso 7 de mayo 2020.

ANDRADE, L. P.; SILVA-ANDRADE, H. M. L.; LYRA-NEVES, R. M.; ALBUQUERQUE, U. P.; TELINOJÚNIOR, W. R. Do artisanal fishers perceive declining migratory shorebird populations? Journal of Ethnobiology and Ethnomedicine, v. 12, n. 16, 2016

ARANGUREN, C. I.; GALEANO, G; BERNAL, R. Manejo actual ael asaí (Eurterpe precatoria Mart.) para la producción de frutos en el sur de la Amazonia Colombiana. Colombia Forestal, v. 17, p. 77-99, 2014.

ATHAYDE, S.; SILVA-LUGO, J.; SCHMINK, M; HECKENBERGER, M. The same, but different: indigenous knowledge retention, erosion, and innovation in the Brazilian Amazon. Human Ecology, v. 45, p. 533-544, 2017.

ATRAN, S.; MEDIN, D.; ROSS, N. Evolution and devolution of knowledge: a tale of two biologies. Journal of the Royal Anthropological Institute, v. 10, p. 395-420, 2004.

AYANTUNDE, A. A.; BRIEJER, M; HIERNAUX, P.; UDO, H. M. J.; TABO, R. Botanical knowledge and its differentiation by age, gender and ethnicity in Southwestern Niger. Human Ecology, v. 36, p. 881-889, 2008.

BAIN, J. Mexican rural women's knowledge of the environment. Mexican Studies, v. 9, p. 259-274, 1993.

BARRERA-BASSOLS, N.; TOLEDO, V. Ethnoecology of the Yucatec Maya: symbolism, knowledge and management of natural resources. Journal of Latin American Geography, v. 4, p. 9-41, 2005.

BEARDSWORTH, A.; BRYMAN, A.; KEIL, T.; GOODE, J.; HASLAM, C.; LANCASHIRE, E. Women, men and food: the significance of gender for nutritional attitudes and choices. British Food Journal, v. 104, p. 
470-491, 2002.

BYERS, E.; SAINJU, M. Mountain ecosystems and women: opportunities for sustainable development and conservation. Mountain Research and Development, v.14, n. 213, 1994.

CABEZAS GONZÁLEZ, A. Anotaciones sobre el tejido feminista latinoamericano: más allá de las genealogías. Contextualizaciones Latinoamericanas, v. 1, p. 1-9, 2015.

CAMOU-GUERRERO, A.; REYES-GARCÍA, V.; MARTÍNEZ-RAMOS, M.; CASAS, A. Knowledge and use value of plant species in a Rarámuri Community: a gender perspective for conservation. Human Ecology, v. 36, p. 259-272, 2007.

CANIAGO, I.; SIEBERT, S. F. Medicinal plant ecology, knowledge and conservation in Kalimantan, Indonesia. Economic Botany, v. 52, n. 3, p. 229-250, 1998

CARDOSO, B.; OCHOA, J.; RICHERI, M.; MOLARES, S.; POZZI, C.; CASTILlO, L.; CHAMORRO, M.; AIGO, J.; MORALES, D.; LADIO, A. Las mujeres y las plantas.la subsistencia de las comunidades rurales de la Patagonia árida. LEISA: Revista de Agroecología, v. 31, p. 20-23, 2015.

CARNEIRO, M. J. Mulheres no campo: notas sobre sua participação política e a condição social do gênero. Estudos Sociedade e Agricultura, v. junio, p. 11-22, 1994.

CARVAlHO, R. M. A.; MARTINS. C. F.; ALVES R. R. N.; ALVES Â. G. C. Do emotions influence the motivations and preferences of keepers of stingless bees? Journal of Ethnobiology and Ethnomedicine, v. 14 , p. $1-11,2018$.

COSTA NETO, E. M.; SANTOS FITA, D.; VARGAS-CLAVIJO, M. Manual de Etnozoología. Primera ed. Valencia: Tundra Ediciones, 2009. 285 p.

CRUZ HERNÁNDEZ, D. T. Una mirada muy otra a los territorios - cuerpos femeninos. Solar Revista de Filosofía Iberoamericana, v. 12, p. 35-46, 2016.

DELGADO, F.; ESCÓBAR, C. Diálogo intercultural e intercientífico para el fortalecimiento de las ciencias de los pueblos indígenas Originarios. Agrupo: Cochabamba, Bolivia. doi: 10.1017/CBO9781107415324.004, 2006.

EDWARDS, S.; NEBEL, S.; HEINRICH, M. Questionnaire surveys: methodological and epistemological problems for field-based ethnopharmacologists. Journal of Ethnopharmacology, v. 100, p. 30-6, 2005.

ESCOBAR, A. Sentipensar con la tierra: las luchas territoriales y la dimensión ontológica de las epistemologías del sur. AIBR, Revista de Antropología Iberoamericana, v. 11, p. 11-32, 2016.

EYSSARTIER, C.; LADIO A. H.; LOZADA, M. Cultural transmission of traditional knowledge in two populations of North-Western Patagonia. Journal of Ethnobiology and Ethnomedicine, v. 4, n. $25,2008$.

EYSSARTIER, C.; LADIO A. H.; LOZADA, M. Horticultural practice and germplasm conservation: a case study in a rural population of the Patagonian steppe. Food Security, v. 7, p. 1259-1271, 2015.

FADIMAN, M. Cultivated food plants: culture and gendered spaces of colonists and the Chachi in Ecuador. Journal of Latin American Geography, v. 4, p. 43-57, 2005.

FORTMANN, L.; ROCHELEAU, D. Women and agroforestry: four myths and three case studies. Agroforesty Systems, v. 2, p. 253-272, 1985.

FURLAN, V.; POCHETTINO M. L.; HILGERT, N. I. Management of fuit species in urban home gardens of Argentina Atlantic Forest as an influence for landscape domestication. Front Plant Sci., v. 8, 2017.

GARIBALDI, A. Moving from model to application: cultural keystone species and reclamation in Fort McKay, Alberta. Journal of Ethnobiology, v. 29, p. 323-338, 2009.

GARIBALDI, A.; TURNER, N. Cultural keystone species: implications for ecological conservation and restoration. Ecology and Society, v. 9, n. 3, 2004. Disponible en: <http://www.ecologyandsociety.org/vol9/iss3/art1>. Acceso en: 25 jun 2020.

GARIBAY-ORIJEL, R.; RAMÍREZ-TERRAZO, A.; ORDAZ-VELÁZQUEZ, M. Women care about local knowledge, experiences from ethnomycology. Journal of Ethnobiology and Ethnomedicine, v. 8, n. 25, p. $1-13,2012$

GASCO, J. 'Le da alegria tener flores' homegardens in the soconusco region of Chiapas, Mexico. Journal of Ethnobiology, v. 28, p. 259-277, 2008.

GOEBEL, A. Process, perception and power: notes from "participatory" research in a Zimbabwean Resettlement Area. Development and Change, v. 29, p. 277-305, 1998.

HERRMANN, T. M.; SCHÜTTLER, E.; BENAVIDES, P.; GÁLVEZ, N., SÖHN, L.; PALOMO, N. Values, animal symbolism, and human-animal relationships associated to two threatened felids in Mapuche and Chilean local narratives. Journal of Ethnobiology and Ethnomedicine, v. 9, n. 41, p. 1-15, 2013.

HOWARD, P. L. Gender and social dynamics in swidden and homegardens in Latin america. In: KUMAR. B. M.; NAIR P. K. R. (ed.).Tropical homegardens: a time tested example of sustainable agroforestry. The Netherlands: Springer Netherlands, 2006. p. 159-182.

HUNN, E. To know them is to love them. Ethnobiology Letters, v. 5, p. 146-150, 2014.

KAI, Z.; WOAN, T. S.; JIE, L.; GOODALE, E.; KITAJIMA, K.; BAGCHI, R.; HARRISON, R. D. Shifting baselines on a tropical forest frontier: extirpations drive declines in local ecological knowledge. PLoS ONE, v. 9, n. 3, 2014. Disponible en: < https://doi.org/10.1371/journal.pone.0092931>. Acceso en: 25 jun 
2020.

KAINER, K.; DURYEA, M.Tapping women's knowledge: plant resource use in extractive reserves, Acre, Brazil. Economic Botany, v. 46, p. 408-425, 1992.

KAY, C. Algunas reflexiones sobre los estudios rurales en América Latina. Íconos - Revista de Ciencias Sociales, v. 1, n. 31, 2013. Disponible en: < https://doi.org/10.17141/iconos.29.2007.230>. Acceso en: 25 jun 2020.

KLIER, G.; NÚÑEZ, P. G. Verde que te quiero verde: una mirada feminista para la conservación de la biodiversidad. Intropica, v. 14, n. 2, p. 171-183, 2019.

KUSHWAH, V. S.; SISODIA, R.; BHATNAGAR, C. Magico-religious and social belief of tribals of district Udaipur, Rajasthan. Journal of Ethnobiology and Ethnomedicine, v. 13, p. 1-7, 2017.

LADIO, A. H. Ethnobiology and research on global environmental change: what distinctive contribution can we make? Ethnobiology and Conservation, v. 6, 2017.

LADIO, A. H.; LOZADA, M. Summer cattle transhumance and wild edible plant gathering in a Mapuche community of Northwestern Patagonia. Human Ecology, v. 32, p. 225-240, 2004.

LEFF, E. Ecofeminismo: el género del ambiente. Polis, v. 9, p. 1-9, 2004.

LEMA, V. S. Criar y ser criados por las plantas y sus espacios en los Andes septentrionales de Argentina. In: BENEDETTI, A.;TOMASI, J. Espacialidades de las tierras altoandinas: avances de investigación desde el Noroeste Argentino. Buenos Aires: Universidad de Buenos Aires, 2013. p. 301-338

LENAERTS, M.; SPADAFORA, A. M. Pueblos indígenas, plantas y mercados: Amazonía y Gran Chaco. FLACSO: Zeta Books, 2008. 210 p.

MAFFI, L. Linguistic, cultural, and biological diversity. Annual Review of Anthropology, v. 34, p. 599-617, 2005.

MAI, Y. H.; MWANGI, E.; WAN, M. Gender analysis in forestry research: looking back and thinking ahead. International Forestry Review, v. 13, p. 245-258, 2012.

NAVARRO TRUJILLO, N.; GUTIÉRREZ AGUILAR, R. Diálogos entre el feminismo y la ecología desde una perspectiva centrada en la reproducción de la vida: entrevista a Silvia Federici. Ecología Política, v. 54, p. $117-120,2017$

NOLAN, J. M.; ROBBINS, M. C.; STAFFORD, J. Emotional meaning and the cognitive organization of ethnozoological domains. Journal of Linguistic Anthropology, v. 11, p. 240-249, 2001.

OCHOA, J. J.; LADIO, A.H. Plantas silvestres con órganos subterráneos comestibles: transmisión cultural sobre recursos subutilizados en la Patagonia (Argentina). Boletin Latinoamericano y del Caribe de Plantas Medicinales y Aromaticas, v. 14, p. 287-300, 2015.

PENA, M. Hacia una voz propia y feminista en el movimiento campesino de Santiago Del Estero. Investigaciones Feministas, v. 8, n. 1, p. 245-266, 2017.

PÉREZ, F. Estado, mujer y hogar en Chile. El Rol Disciplinario Época. Revista Austral de Ciencias Sociales, v. 37, p. 29-48, 2019.

PRICE, L. L.; OGLE, B. M. Gathered indigenous vegetables in mainland southeast asia: a gender asset. gender and natural resource management: livelihoods, mobility and interventions. New York, USA: Earthscan, 2008. 65 p.

PULEO, A. H. Ecología y género en diálogo interdisciplinar. Primera ed. Madrid: Plaza yValdes Editores, 2015. $414 \mathrm{p}$.

REYES-GARCÍA, V.; BROESCH, J.; CALVET-MIR, L.; FUENTES-PELÁEZ, N.; MCDADE, T. W.; PARSA, S.; TANNER, S.; HUANCA, T.; LEONARD, W. R.; MARTÍNEZ-RODRÍGUEZ, M. R. Cultural transmission of ethnobotanical knowledge and skills: an empirical analysis from an amerindian society. Evolution and Human Behavior, v. 30, p. 274-285, 2009.

REYES-GARCÍA, V.; VILA, S.; ACEITUNO-MATA, L.; CALVET-MIR, L.; GARNATJE, T.; JESCH, A.; LASTRA, J. J.; PARADA, M.; RIGAT, M.; VALLÈS, J.; PARDO-DE-SANTAYANA, M. Gendered homegardens: a study in three mountain areas of the Iberian Peninsula. Economic Botany, v. 64, p. 235247, 2010.

RICHERI, M.; CARDOSO, M. B.; LADIO, A. H.. Soluciones locales y flexibilidad en el conocimiento ecológico tradicional frente a procesos de cambio ambiental: Estudios de caso en Patagonia. Ecología Austral, v. 23, n. 3, p. 184-193, 2013.

ROZZI, R. Biocultural ethics: recovering the vital links between the inhabitants, their habits, and habitats. Environmental Ethics, v. 34, p. 27-50, 2012.

ROZZI, R. Bioética global y ética biocultural. Cuadernos de Bioética, v. 27, p. 339-355, 2016.

RUIZ PÉREZ, L. M.; ARGUETA VILlAMAR, A. Saberes indígenas y diálogo intercultural. Cultura Científica y Saberes Locales, v. 5, p. 31-56, 2011.

SHAHID, S.; BLEAM, R.; BESSARAB, D.; THOMPSON, S. C. 'If you don't believe it, it won't help you"': use of bush medicine in treating cancer among aboriginal people in Western Australia. Journal of Ethnobiology and Ethnomedicine, v. 6, p. 1-9, 2010.

SHIVA, V. Abrazar la vida. Primera ed. Montevideo: Instituto del Tercer Mundo, 1991. 264 p. 
SILIPRANDI, E. Mujeres y agroecología. nuevos sujetos políticos en la agricultura familiar. Investigaciones Feministas, v. 1, p. 125-137, 2010.

SUÁREZ, M. E. El análisis de narrativas en etnobotánica: el "yuchán” (Ceiba chodatii, Bombacaceae) en el discurso de los Wichís del Chaco Semiárido Salteño, Argentina. Bol. Soc. Argent., v. 44, p. 405-419, 2009.

TASSI, É. M. M.; BEZERRA, I. A Soberania alimentar que desperta e aprofunda os saberes em direitos por terra, por comida de verdade e por igualdade de gênero. Revista em Extensão, v. 1, maio, p. 42-52, 2020.

TOLEDO, V.; ALARCÓN-CHÁIRES, P. Tópicos bioculturales: reflexiones sobre el concepto de bioculturalidad y la defensa del patrimonio biocultural de México. Primera ed. Morelia: Universidad Autónoma de México, 2018. 120 p.

TOLEDO, V. M.; BARRERAS-BASSOLS, N. La memoria biocultural: la importancia agroecológica de las sabidurías tradicionales. Primera ed. Barcelona: Icaria editorial, 2008. 230 p.

TORRES-AVILEZ, W.; DE MEDEIROS, P. M.; ALBUQUERQUE, U. P. Effect of gender on the knowledge of medicinal plants: systematic review and meta-analysis. Evidence-based Complementary and Alternative Medicine, v. 2016, p. 12-15, 2016.

TORRI, M. C. Medicinal plants used in mapuche traditional medicine in Araucania, Chile: linking sociocultural and religious values with local heath practices. Complementary Health Practice Review, v. 15, p. 132$148,2010$.

TRPIN, V.; RODRIGUEZ, M. D.; BROUCHOUD, S. desafíos en el abordaje del trabajo rural en el norte de la Patagonia: Mujeres en forestación, horticultura y fruticultura. Trabajo y Sociedad, v. 28, p. 267-280, 2017.

ULlOA, A. Feminismos territoriales en América Latina: defensas de la vida frente a los extractivismos. Nomadas, v. 45, p. 123-139, 2016.

VARGAS-CLAVIJO, M.; MEDEIROS, E.; COSTA NETO, E. M. Actitudes hacia la fauna: algunas explicaciones de la conducta humana hacia los animales. In: ALVES, R. R.; SOUTO, W. M.; MOURÃO, J. (ed.). A Etnozoologia no Brasil: importancia, status atual e perspectivas. Recife: Nupeea, 2010. p. 1-23.

VÁRI, Á.; ARANY, I.; KALÓCZKAI, Á.; KELEMEN, K.; PAPP, J.; CZÚCZ, B. Berries, greens, and medicinal herbs - mapping and assessing wild plants as an ecosystem service in Transylvania (Romania). Journal of Ethnobiology and Ethnomedicine, v. 16, p. 1-14, 2020.

VAZQUEZ-GARCIA, V. Gender, ethnicity, and economic status in plant management: uncultivated edible plants among the Nahuas and Popolucas of Veracruz, Mexico. Agriculture and Human Values, v. 25, p. 65-77, 2007.

VELASCO SESMA, A. Género y valores: aportaciones de las mujeres al debate sobre la consideración moral. Dilemata, v. 7, p. 259-279, 2015.

VIEIRA, B. B.; MILWARD-DE-AZEVEDO, M. A. A etnobotânica e o ecofeminismo em prol da conservação ambiental. Diversidade e Gestão, v. 2, p. 178-188, 2018.

VILlAMAR, A. A.; DIAGO, O. L. S.; CONTRERAS, E. J. C.; MEDINACELI, A. Código de ética para la investigación etnobiológica en América Latina. Ethnoscientia, v. 3, 2018.

VIU, A.; VIU, M.; CAMPOS, L. 2010. Etnobotânica: uma questão de gênero? Revista Brasileira de Agroecologia, v. 5, p. 138-147, 2010.

VOEKS, R. A. Are women reservoirs of traditional plant knowledge? Gender, ethnobotany and globalization in Northeast Brazil. Singapore Journal of Tropical Geography, v. 28, p. 7-20, 2007.

VOGL, C. R.; VOGL-LUKASSER, B.; PURI, R. K. Tools and methods for data collection in ethnobotanical studies of homegardens. Field Methods, v. 16, p. 285-306, 2004.

WESTERMANN, O.; ASHBY, J.; PRETTY, J. Gender and social capital: the importance of gender differences for the maturity and effectiveness of natural resource management groups. World Development, v. 33, p. 1783-1799, 2005.

ZENT, E. Ecogonía III. Jkyo ecogonía III. Jkyo jkwainï: la filosofía del cuidado de la vida de los Jotï del Amazonas Venezolano. Etnoecológica, v. 10, n. 8, p. 1-28, 2014. 